\title{
Circulating tumor cells as a potential biomarker for postoperative clinical outcome in HBV-related hepatocellular carcinoma
}

This article was published in the following Dove Press journal:

Cancer Management and Research

\section{Xinping $\mathrm{Ye}^{1, *}$ \\ Guanghui Li ${ }^{1, *}$ \\ Chuangye Han' \\ Quanfa Han' \\ Liming Shang' \\ $\mathrm{Hao} \mathrm{Su}{ }^{\prime}$ \\ Bowen Han' \\ Yizhen Gong' \\ Guodong Lu ${ }^{2}$ \\ Tao Peng'}

'Department of Hepatobiliary Surgery, The First Affiliated Hospital of Guangxi Medical University, Nanning, Guangxi Province, China; ${ }^{2}$ Department of Toxicology, School of Public Health, Guangxi Medical University, Nanning, Guangxi Province, China

*These authors contributed equally to this work
Correspondence: Tao Peng Department of Hepatobiliary Surgery, First Affiliated Hospital of Guangxi Medical University, 6 Shuangyong Road, Nanning 53002I, Guangxi Province, China

Tel +867715356528

Fax +86 77I 535003 I

Email pengtaogmu@।63.com
Background: This study aimed to determine if the number of circulating tumor cells (CTCs) and changes in their numbers affected tumor recurrence and metastasis after surgical resection in patients with hepatitis B virus (HBV)-related hepatocellular carcinoma (HCC).

Methods: The primary endpoints were overall survival (OS) and progression-free survival (PFS). A total of 42 patients with HCC were selected from the First Affiliated Hospital of Guangxi Medical College from 2014 to 2017. CTCs were counted 1 day prior to and 30 days after surgical excision of HCC using the CanPatrol $^{\mathrm{TM}}$ system.

Results: Numbers of CTCs (> 2 CTCs and > 5 CTCs per $5 \mathrm{ml}$ peripheral blood) were significantly associated with Edmondson stage in HBV-related HCC prior to surgery ( $P=0.004$ and 0.014 , respectively). However there were no significant associations between other tested clinicopathological factors and CTC counts. Postoperative CTC counts $(>2$ and $>5)$ and pre/postoperative change in CTC counts were significantly associated with PFS ( $P=0.02,0.009$, and 0.001 , respectively), but not with OS. Receiver operating characteristic curve analysis showed that pre/postoperative changes in the CTC count were a better predictor of performance than absolute count. The postoperative CTC count was also significantly associated with positive TP53 expression $(P<0.05)$. Conclusion: These results demonstrate that postoperative CTC counts ( $>2$ and $>5$ ) and changes in CTC counts may be independent prognostic indicators for PFS in patients with HBV-related $\mathrm{HCC}$, with the change in number of CTCs showing better predictive performance.

Keywords: circulating tumor cell, hepatocellular carcinoma, progression-free survival, overall survival

\section{Introduction}

Hepatocellular carcinoma (HCC) is one of the most frequently diagnosed cancers and the third most common cause of cancer-related deaths worldwide. ${ }^{1}$ Although surgical resection is the preferred therapeutic choice for $\mathrm{HCC}$, the overall prognosis of patients with liver cancer remains poor, with an estimated 5 -year survival rate of only $12.7 \%{ }^{2}$ mainly due to recurrence and metastasis after resection. ${ }^{3,4}$ The recent increase in precision medicine has led to an international consensus suggesting that individual liver cancer patients should be treated differently to maximize their chance of survival. However, lack of sensitive and specific indicators for detecting early metastasis and recurrence of liver cancer means that these may not be detected early enough, and many patients may thus miss the effective treatment periods. Consequently, the 3 - and 5-year survival rates of liver cancer patients are still not optimistic. ${ }^{5}$ It is therefore necessary to identify and employ novel biomarkers to monitor the prognosis of HCC patients more effectively. 
Recent studies have shown that circulating tumor cells (CTCs) can provide a dynamic, easily available body-fluid biopsy specimen. ${ }^{6,7}$ The concept of CTCs was initially proposed in the 1870s by an Australian scholar who suggested that "circulating tumor cells" were cells similar to those of the primary tumor found in the blood of metastatic cancer patients. ${ }^{8} \mathrm{CTCs}$ originate from primary tumors and spread to distant sites, but are mostly eliminated by immune cells such as natural killer cells. Although very few cancer cells thus survive in the circulatory system, these may then form small tumor metastases under certain conditions. Several studies have indicated that CTCs represent an intermediate process of the formation of metastatic lesions and are an independent risk factor associated with the prognosis of solid tumors. ${ }^{9}$ Isolating and detecting CTCs will therefore help us to understand the processes of early metastasis and recurrence and tumor aggressiveness, ${ }^{10}$ which have been major areas of interest in the field of cancer research since CTCs were confirmed to exist in various types of cancer. Recent research has shown that tumor patients with positive CTC counts often have a poor prognosis, including in terms of tumor-free survival and overall survival (OS), while timely monitoring of CTCs in HCC patients can be a good predictor of clinical efficacy, disease progression, and patient prognosis. ${ }^{11-13}$ However, the relationships between CTC counts and tumor metastasis, invasion, and prognosis in patients with hepatitis $\mathrm{B}$ virus (HBV)-related HCC remain unclear. We previously isolated and tested CTCs using CanPatrol ${ }^{\mathrm{TM}}$ CTC-detection technology, which has been confirmed to isolate and test CTCs effectively. ${ }^{14}$ The aim of the current study was to determine the correlations between the number of CTCs and changes in their numbers, and clinical features and postoperative clinical outcomes in patients with HBV-related HCC.

\section{Materials and methods Study population and design}

This study enrolled 42 patients with HBV-related HCC in the First Affiliated Hospital of Guangxi Medical University (Nanning, China) from December 2014 to February 2017. Their diagnosis was consistent with the Treatment Specification of Primary Liver Cancer (2011 Edition). ${ }^{15}$ The study was approved by the medical ethical committees of the First Affiliated Hospital of Guangxi Medical College, and all blood samples were obtained with written informed consent from the patients. The inclusion criteria were: a confirmed pathological diagnosis of HCC; $\geq 18$ and $\leq 70$ years old; no prior systemic medical therapy for cancer and World Health Organization performance status of 0-2; laparoscopic tumor resection or laparotomy; generally good health status, with no obvious lesions of the heart, lung, kidney, or other vital organs; and complete medical records available. Patients were excluded if they had any other previous malignancy or severe organ dysfunction, or if their medical records were incomplete. The number of CTCs was measured before and 1 month after surgery, and the patients were divided into two groups based on the number of CTCs $(\leq 2$ and $>2)$ per $5 \mathrm{~mL}$ of peripheral blood. Clinical data including gender, age, alpha-fetoprotein (AFP), HBV-DNA, metastasis, TNM staging, recurrence, and metastasis were compared between the two groups.

\section{Immunostaining and scoring}

All patients underwent surgery to resect the tumors and postoperative specimens were sent for pathological examination. Samples including the tumor and surrounding tissue were fixed in formaldehyde, dehydrated in graded ethanols, embedded in paraffin, and cut into $4-\mu \mathrm{m}$ slices for immunostaining. Immunostaining was carried out using the enhanced labeled polymer system with PBS as a negative control instead of primary antibody. Antigen retrieval was performed by microwave heating in citrate buffer. The remaining steps were carried out according to the reagent instructions. p21, NM23, CD34, TP53, and vascular endothelial growth factor (VEGF) antibody reagents were all purchased from Maixin Company (Fuzhou, China). Light yellow, yellow, and brown staining were all considered as positive staining results. We examined the cell-staining intensities and percentages of positive cells in three random high-power fields $(\times 400)$. For p53, NM23, VEGF, and $\mathrm{p} 21$, an average of $<5 \%$ high-magnification positive cells was defined as negative (-), $5 \%-25 \%$ as,$+ 26 \%-50 \%$ as ++ , and $>50 \%$ as +++ . CD34 immunostaining was used to identify sinusoids at $\times 200$ magnification. The average number of CD34-positive sinusoids was taken as the standard, with greater numbers indicating high-density blood vessels and lower numbers indicating low-density blood vessels.

\section{Isolation and enumeration of CTCs}

Peripheral blood samples $(5 \mathrm{~mL})$ were collected from HCC patients by venipuncture into EDTA tubes. The required filtration system included a filtration tube containing a membrane (SurExam, Guangzhou, China).

We established three types of nucleic acid probes to identify and examine the expression levels of epithelial and mesenchymal genes in CTCs by multiplex RNA in situ hybridization assay. The CTCs were then further typed by examining the different colors of fluorescence signals under 
a fluorescence microscope, with red and green fluorescence representing epithelial- and mesenchymal-type gene expression, respectively. Hybrid CTCs included both red and green fluorescence.

\section{Follow-up}

The 42 patients were followed up through outpatient review and telephone, with a final follow-up date of April 30, 2017. Progression-free survival (PFS) was defined as the time from blood collection until disease progression, death, or the day of the last follow-up visit, whichever came first. OS was defined as the time from blood collection until the date of death from any cause, or the most recent documented follow-up visit.

\section{Statistical analysis}

All statistical evaluations and analyses were performed using SPSS software version 17.0 (SPSS Inc., Chicago, IL, USA) or GraphPad Prism 5.0 (GraphPad Software, Inc., La Jolla, CA, USA). The associations between CTC positivity and clinicopathological characteristics and immunostaining results were analyzed by $\chi^{2}$ or Fisher's exact tests using SPSS, and survival analyses were performed using GraphPad Prism 5.0. Kaplan-Meier survival curves were used to analyze the univariate distributions for PFS and OS. Crude or adjusted hazard ratios (HRs) and 95\% CI were calculated by univariate and multivariate analyses by Cox proportional hazards regression analysis. Statistical significance was based on a two-sided significance level of 0.05 .

\section{Results}

\section{Patient characteristics and clinical outcomes}

A total of $42 \mathrm{HCC}$ patients (37 males, five females; median age 47 years, range $27-68$ years) were enrolled in this study. All patients were Child-Pugh class A. The characteristics of the enrolled patients in terms of age, gender, AFP, HBV-DNA, Edmondson stage, TNM stage, portal vein tumor thrombosis, microsatellite damage, postoperative recurrence, metastasis, tumor number, and tumor size are summarized in Table 1. Preoperative CTC counts were significantly associated with Edmondson stage ( $>2$ CTCs and $>5$ CTCs, $P=0.044$ and 0.014, respectively). However, there were no significant associations between any other clinicopathological features and CTC counts.

Ten patients received laparoscopic tumor resection and the remaining 32 received laparotomy. There was no significant association between surgical approach and baseline CTC counts $(P>0.05)$.

\section{Associations between CTCs and immunohistochemical markers}

We investigated the associations between commonly used clinical immunohistochemical markers and CTCs. Postoperative CTC counts were significantly associated with positive p53 expression $(P<0.05$; Table 1$)$. TP53 mutations have been shown to result in positive expression of $\mathrm{p} 53$, and we therefore inferred that tumor cells with TP53 mutation were more likely to enter the circulation during tumor resection. However, further studies are needed to verify this hypothesis.

\section{Associations between CTCs and clinical outcomes in HBV-related HCC patients} The median number of CTCs in $5 \mathrm{~mL}$ of peripheral blood detected preoperatively was four (range $0-31$ ), and the median number detected postoperatively was three (0-22). Thirty-three of the 42 patients had preoperative CTCs and 34 had postoperative CTC counts $\geq 1$, with positivity rates of $78.57 \%$ and $80.95 \%$, respectively. Notably, the postoperative CTC count increased in 17 and decreased in 25 cases. We also analyzed the association between CTC counts and postoperative clinical outcomes and found no significant associations between postoperative CTC counts and PFS or OS ( $P>0.05$; Figure 1, Table 2). However, patients with a postoperative CTC counts $\leq 2$ or $\leq 5$ had significantly longer PFS than patients with postoperative CTC counts $>2$ or $>5$ ( $P=0.0136$ and $P=0.0018$, respectively; Figure 2). Patients with postoperative CTC counts $>5$ also had poorer OS than those with postoperative $\mathrm{CTC}$ counts $\leq 5$ ( $P=0.012$; Figure 2 ). Furthermore, patients with a postoperative increase in CTCs had significantly longer median PFS and OS compared with patients with a postoperative decrease in CTCs $(P<0.0001$ and $P=0.021$, respectively; Figure 3 ).

We analyzed the associations between CTC counts and postoperative clinical outcomes using Cox proportional hazard regression models (Table 3 ), which demonstrated that postoperative CTC counts ( $>2$ and $>5$ ) and changes in CTC counts were significantly associated with PFS, but not with OS. This suggested that postoperative CTC counts ( $>2$ and $>5$ ) and changes were independent prognostic indicators for PFS in patients with HBV-related HCC. We investigated the predictive powers of these indicators by ROC analysis and showed that the pre/postoperative change in CTC counts was a better predictor than postoperative CTC counts (area under the curve $0.732,0.732$, and 0.893 ; sensitivity 0.571 , 0.857 , and $0.929 ; 1$-specificity $0.393,0.107$, and 0.143 , respectively). 
Table I Associations between CTCs and clinical data and immunohistochemical markers

\begin{tabular}{|c|c|c|c|c|c|c|c|c|c|c|c|c|c|c|c|}
\hline \multirow[t]{2}{*}{ Type } & \multicolumn{2}{|c|}{$\begin{array}{l}\text { Pre- } \\
\text { CTCs }\end{array}$} & \multirow[t]{2}{*}{$P$-value } & \multicolumn{2}{|c|}{$\begin{array}{l}\text { Pre- } \\
\text { CTCs }\end{array}$} & \multirow[t]{2}{*}{$P$-value } & \multicolumn{2}{|c|}{$\begin{array}{l}\text { Post- } \\
\text { CTCs }\end{array}$} & \multirow[t]{2}{*}{$P$-value } & \multicolumn{2}{|c|}{$\begin{array}{l}\text { Post- } \\
\text { CTCs }\end{array}$} & \multirow[t]{2}{*}{$P$-value } & \multicolumn{2}{|c|}{ Change-CTCs } & \multirow[t]{2}{*}{$P$-value } \\
\hline & $\leq 2$ & $>2$ & & $\leq 5$ & $>5$ & & $\leq 2$ & $>2$ & & $\leq 5$ & $>5$ & & Reduce & Rise & \\
\hline Gender & & & 0.611 & & & 0.088 & & & 0.731 & & & $>0.999$ & & & 0.139 \\
\hline Male & 16 & 21 & & 26 & II & & 15 & 22 & & 27 & 10 & & 20 & 17 & \\
\hline Female & 1 & 4 & & 1 & 4 & & 3 & 2 & & 4 & 1 & & 5 & 0 & \\
\hline AFP (ng/mL) & & & 0.804 & & & 0.879 & & & 0.554 & & & $>0.999$ & & & 0.804 \\
\hline$<400$ & 13 & 17 & & 20 & 10 & & 12 & 18 & & 22 & 8 & & 17 & 13 & \\
\hline$\geq 400$ & 4 & 8 & & 7 & 5 & & 6 & 6 & & 9 & 3 & & 8 & 4 & \\
\hline Age (years) & & & 0.324 & & & 0.85 & & & 0.463 & & & 0.222 & & & 0.024 \\
\hline$\geq 47$ & 8 & 8 & & 10 & 6 & & 8 & 8 & & 14 & 2 & & 13 & 3 & \\
\hline$<47$ & 9 & 17 & & 17 & 9 & & 10 & 16 & & 17 & 9 & & 12 & 14 & \\
\hline HBV-DNA & & & 0.276 & & & 0.353 & & & 0.15 & & & 0.389 & & & 0.037 \\
\hline$<5.0 \times 10^{2}$ & 9 & 9 & & 13 & 5 & & 10 & 8 & & 15 & 3 & & 14 & 4 & \\
\hline$\geq 5.0 \times 10^{2}$ & 8 & 16 & & 14 & 10 & & 8 & 16 & & 16 & 8 & & 11 & 13 & \\
\hline Edmondson stage & & & 0.044 & & & 0.014 & & & 0.353 & & & 0.060 & & & 0.542 \\
\hline I, II & 14 & 13 & & 21 & 6 & & 13 & 14 & & 23 & 4 & & 17 & 10 & \\
\hline III, IV & 3 & 12 & & 6 & 9 & & 5 & 10 & & 8 & 7 & & 8 & 7 & \\
\hline TNM stage & & & 0.511 & & & 0.478 & & & $>0.999$ & & & 0.836 & & & 0.152 \\
\hline I, II & 15 & 22 & & 25 & 12 & & 16 & 21 & & 28 & 9 & & 24 & 13 & \\
\hline III, IV & 2 & 3 & & 2 & 3 & & 2 & 3 & & 3 & 2 & & 1 & 4 & \\
\hline BCLC stage & & & 0.312 & & & 0.666 & & & 0.100 & & & $>0.999$ & & & 0.834 \\
\hline$A, B$ & 12 & 22 & & 20 & 14 & & 12 & 22 & & 25 & 9 & & 21 & 13 & \\
\hline C, D & 5 & 3 & & 7 & 1 & & 6 & 2 & & 6 & 2 & & 4 & 4 & \\
\hline PVTT & & & 0.158 & & & 0.53 & & & 0.178 & & & 1.00 & & & $>0.999$ \\
\hline Yes & 2 & 0 & & 2 & 0 & & 2 & 0 & & 2 & 0 & & I & I & \\
\hline No & 15 & 25 & & 25 & 15 & & 16 & 24 & & 29 & 11 & & 24 & 16 & \\
\hline Tumor size (cm) & & & $>0.999$ & & & $>0.999$ & & & 0.554 & & & $0.78 I$ & & & 0.655 \\
\hline$>5$ & 5 & 7 & & 8 & 4 & & 6 & 6 & & 8 & 4 & & 6 & 6 & \\
\hline$\leq 5$ & 12 & 18 & & 19 & 11 & & 12 & 18 & & 23 & 7 & & 19 & 11 & \\
\hline Number of tumors & & & 0.160 & & & $>0.999$ & & & 0.676 & & & 0.754 & & & 0.160 \\
\hline Singleton & 12 & 23 & & 22 & 13 & & 16 & 19 & & 25 & 10 & & 23 & 12 & \\
\hline Multiple & 5 & 2 & & 5 & 2 & & 2 & 5 & & 6 & 1 & & 2 & 5 & \\
\hline P2I & & & $0.86 \mathrm{I}$ & & & $>0.999$ & & & $0.95 I$ & & & $>0.999$ & & & $0.86 I$ \\
\hline Positive (+) & 3 & 3 & & 4 & 2 & & 3 & 3 & & 4 & 2 & & 3 & 3 & \\
\hline Negative (-) & 12 & 21 & & 19 & 12 & & 12 & 19 & & 25 & 8 & & 21 & 12 & \\
\hline NM23 & & & $>0.999$ & & & 0.375 & & & $>0.999$ & & & 0.275 & & & 0.400 \\
\hline Positive (+) & 15 & 24 & & 25 & 14 & & 16 & 23 & & 29 & 10 & & 24 & 15 & \\
\hline Negative (-) & 0 & 1 & & 0 & 1 & & 0 & 1 & & 0 & 1 & & 0 & 1 & \\
\hline VEGF+ & & & 0.332 & & & 0.945 & & & 0.501 & & & 0.525 & & & 0.987 \\
\hline Positive (+) & 7 & 15 & & 14 & 8 & & 8 & 14 & & 15 & 7 & & 13 & 9 & \\
\hline Negative (-) & 8 & 9 & & 11 & 6 & & 8 & 9 & & 14 & 3 & & 10 & 7 & \\
\hline CD34 & & & $>0.999$ & & & 0.385 & & & $>0.999$ & & & $>0.999$ & & & $>0.999$ \\
\hline Positive (+) & 15 & 23 & & 24 & 14 & & 15 & 23 & & 27 & 11 & & 21 & 17 & \\
\hline Negative (-) & 0 & 1 & & 0 & 1 & & 0 & I & & 1 & 0 & & 1 & 0 & \\
\hline
\end{tabular}

Note: Bold values indicate statistically significant values.

Abbreviations: CTC, circulating tumor cell; BCLC, Barcelona Clinic Liver Cancer; HBV-DNA, hepatitis B virus DNA; AFP, alpha-fetoprotein; VEGF, vascular endothelial growth factor; PVTT, portal vein tumor thrombosis.

\section{Discussion}

CTCs exist in the peripheral circulation of cancer patients. Tumors were established by injecting human HCC cells into the Glisson sheath of immunocompromised (NOD/SCID) mice, and CTCs released from the primary tumor site were then continuously detected in the circulating blood. ${ }^{16}$ Furthermore,
CTCs have similar properties to the primary lesions and can therefore be used as "liquid live-test specimens," providing convenient access to tumor cells and earlier diagnosis of potentially fatal metastases. ${ }^{17}$ CTCs can be isolated and enriched from a small volume of peripheral blood, with minimal patient trauma.${ }^{6} \mathrm{CTC}$ also have a greater specificity and accuracy for 
A

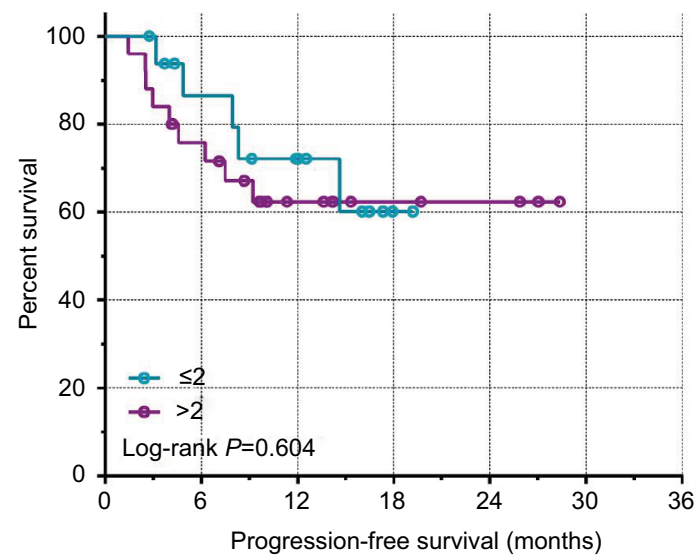

C

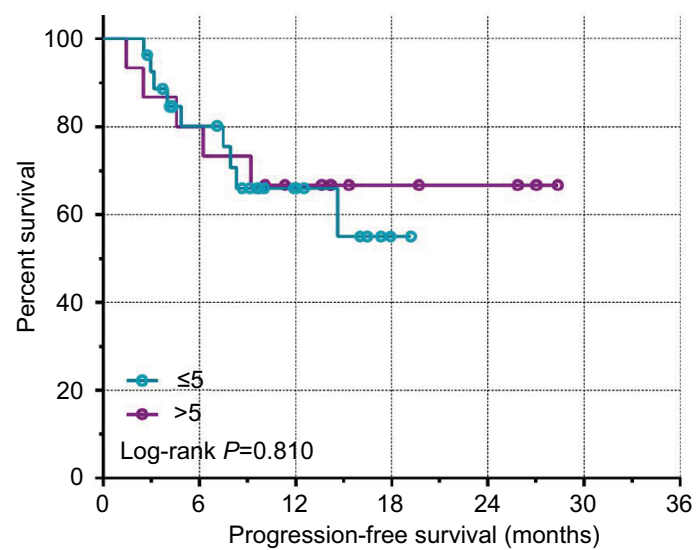

B

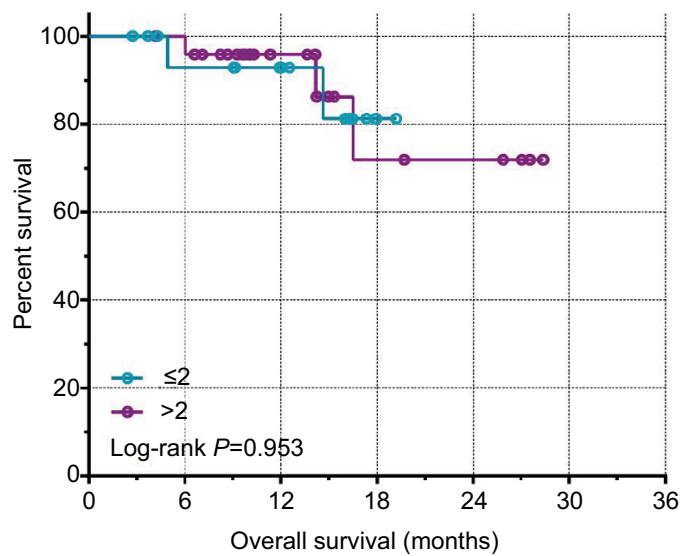

D

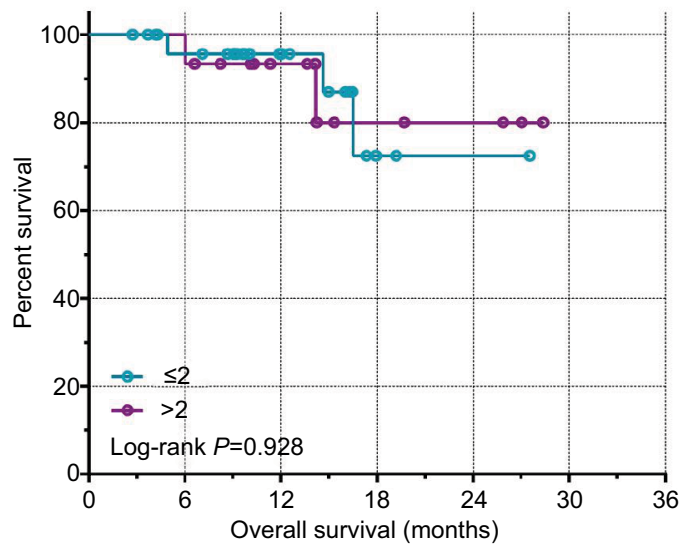

Figure I Kaplan-Meir survival curves for preoperative CTC counts.

Notes: (A) PFS with a CTC count cut-off of 2. (B) OS with a CTC count cut-off of 2. (C) PFS with a CTC count cut-off of 5 . (D) OS with CTC count cut-off of 5. Abbreviations: CTC, circulating tumor cell; PFS, progression-free survival; OS, overall survival.

detecting HCC compared with the traditional marker, AFP. However, their rarity in the peripheral blood means that CTCs are difficult to detect. Although numerous techniques have recently been used to test CTCs, only a few have been used to enrich and detect CTCs from HCC patients, including density gradient centrifugation, PCR, or quantitative real-time PCR (qRT-PCR), isolation by size of epithelial tumor cells, flow cytometry, and magnetic cell fractionation. However, many techniques are associated with problems including poor purity, lower cover rate, and a time-consuming nature, which have reduced the accuracy of CTC detection. In the current study, we isolated and tested CTCs using the CanPatrol ${ }^{\mathrm{TM}}$ system, which is based on qRT-PCR. qRT-PCR can improve the sensitivity and specificity of CTC detection and can reduce false-negative and false-positive results by analyzing the expression of multiple genes simultaneously. ${ }^{18}$ Compared with other detection techniques, CanPatrol ${ }^{\mathrm{TM}}$ is a second-generation CTC-enrichment technique based on immune removal combined with nanofil- tration technology, which does not rely on specific antibody capture and removal of white blood cells. It has demonstrated an efficiency of $>99.97 \%$ and a tumor-cell recovery rate of $>80 \% .{ }^{14} \mathrm{CanPatrol}^{\mathrm{TM}}$ can isolate and enrich CTCs, including those with the most metastatic potential (CTCs- circulating tumor microembolis) from small blood samples $(5 \mathrm{~mL})$, which is critical for monitoring tumor recurrence and for providing noninvasive samples for molecular detection.

Several studies have indicated that CTCs are an independent risk factor closely associated with tumor infiltration, portal vein thrombosis, Child-Pugh class grade B/C level, and the prognosis of HCC. ${ }^{6}$ Patients with higher CTC counts may have poorer outcomes, higher recurrence risks, and lower disease-free survival and OS after surgery. Sun et al ${ }^{19}$ examined CTC counts in preoperative blood samples from $123 \mathrm{HCC}$ patients with long-term follow-up and found that the risk of early postoperative recurrence was significantly higher in patients with $\geq 2$ preoperative CTCs per $7.5 \mathrm{~mL}$ 
Table 2 Associations between clinical data and clinical outcomes

\begin{tabular}{|c|c|c|c|c|c|}
\hline \multirow[t]{2}{*}{ Variables } & \multirow{2}{*}{$\begin{array}{l}\text { Patients } \\
(n=42)\end{array}$} & \multicolumn{2}{|c|}{ Overall survival } & \multicolumn{2}{|c|}{ Progress-free survival } \\
\hline & & Chi square & P-value* & Chi-square & P-value* \\
\hline Gender & & 1.56 & 0.212 & 2.97 & 0.085 \\
\hline Male & 37 & & & & \\
\hline Female & 5 & & & & \\
\hline Age (years) & & 0.256 & 0.213 & 2.323 & 0.127 \\
\hline$\geq 50$ & 16 & & & & \\
\hline$<50$ & 26 & & & & \\
\hline AFP (ng/mL) & & 0.186 & 0.666 & 0.156 & 0.693 \\
\hline$<400$ & 30 & & & & \\
\hline$\geq 400$ & 12 & & & & \\
\hline HBV-DNA & & 0.119 & 0.730 & 0.289 & 0.591 \\
\hline$<5.0 \times 10^{2}$ & 18 & & & & \\
\hline$\geq 5.0 \times 10^{2}$ & 24 & & & & \\
\hline Edmondson stage & & 2.702 & 0.10 & 0.9784 & 0.323 \\
\hline I, II & 27 & & & & \\
\hline III, IV & 15 & & & & \\
\hline TNM stage & & 13.5 & $0.0002 *$ & 6.546 & 0.0105 \\
\hline I, II & 37 & & & & \\
\hline III, IV & 5 & & & & \\
\hline BCLC stage & & 1.091 & 0.2964 & 0.08222 & 0.774 \\
\hline$A, B$ & 34 & & & & \\
\hline C, D & 8 & & & & \\
\hline Diameter of tumors $(\mathrm{cm})$ & & 2.829 & 0.093 & 0.0611 & 0.805 \\
\hline$\leq 5$ & 30 & & & & \\
\hline$>5$ & 12 & & & & \\
\hline Number of tumors & & 2.531 & 0.112 & 4.682 & $0.031 *$ \\
\hline Single & 35 & & & & \\
\hline Multiple tumors & 7 & & & & \\
\hline Pre-CTCs & & 0.00344 & 0.953 & 0.2687 & 0.604 \\
\hline$\leq 2$ & 17 & & & & \\
\hline$>2$ & 25 & & & & \\
\hline Pre-CTCs & & & & & \\
\hline$\leq 5$ & 27 & 0.008 & 0.928 & 0.058 & 0.809 \\
\hline$>5$ & 15 & & & & \\
\hline Post-CTCs & & 0.516 & 0.814 & 8.37 & $0.0136 *$ \\
\hline$\leq 2$ & 18 & & & & \\
\hline$>2$ & 24 & & & & \\
\hline Post-CTCs & & 6.601 & $0.0122 *$ & 9.744 & $0.0018^{*}$ \\
\hline$\leq 5$ & 31 & & & & \\
\hline$>5$ & 11 & & & & \\
\hline Post-CTC changes & & 4.819 & $0.021^{*}$ & 19.24 & $<0.0001 *$ \\
\hline Reduce & 25 & & & & \\
\hline Rise & 17 & & & & \\
\hline
\end{tabular}

Notes: ${ }^{* P}$-values based on log-rank tests.

Abbreviations: AFP, alpha-fetoprotein; HBV-DNA, hepatitis B virus DNA; BCLC, Barcelona Clinic Liver Cancer; CTC, circulating tumor cell.

compared with those with $<2$ per $5 \mathrm{~mL}(P<0.0001)$. CTCs were detected in the peripheral blood in 33 of the 42 patients (78.6\%) in the current study, which was similar to a previous report of $80 \% .{ }^{20}$ Kelley et $\mathrm{l}^{6}$ detected CTCs in the peripheral blood of $20 \mathrm{HCC}$ patients and found significant associations between CTC counts per $7.5 \mathrm{~mL}$ peripheral blood and AFP values. In contrast, we found no significant associations between CTC counts and any of the tested clinicopathological features, except Edmondson stage. CTC count ( $>2$ or $\leq 2$ per $5 \mathrm{~mL}$ ) was therefore not significantly associated with recurrence or metastasis. This may have been because the sample size was too small, because of differences in techniques and/ or experience among the surgeons, or the results may have been affected by whether or not the patient was actively 
A

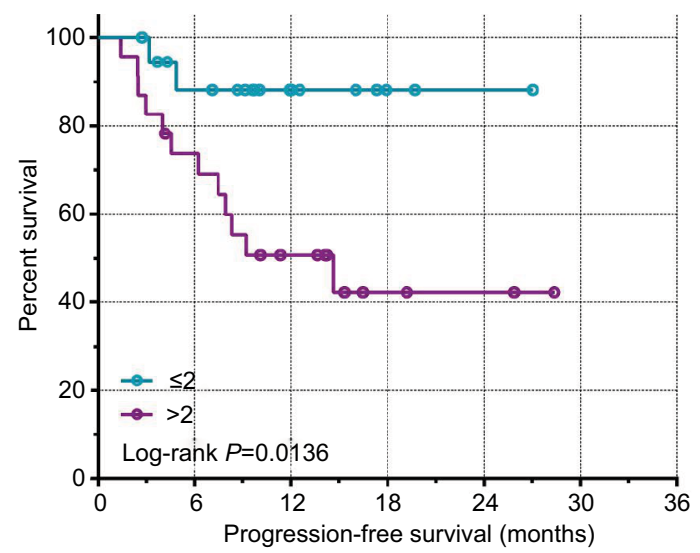

C

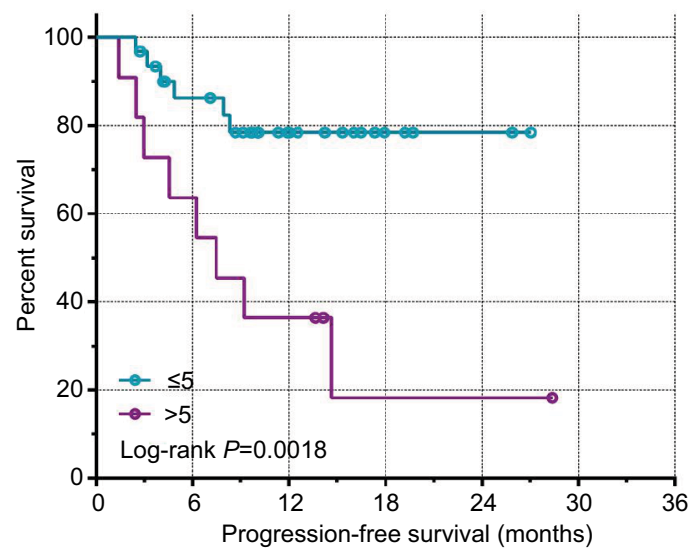

B

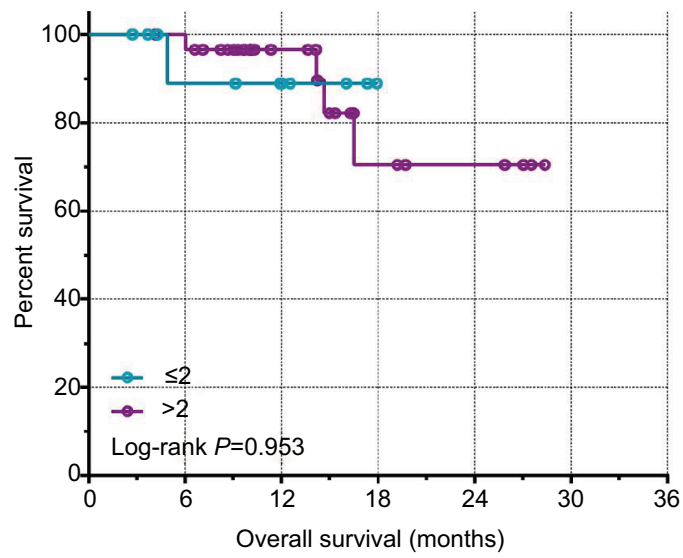

D

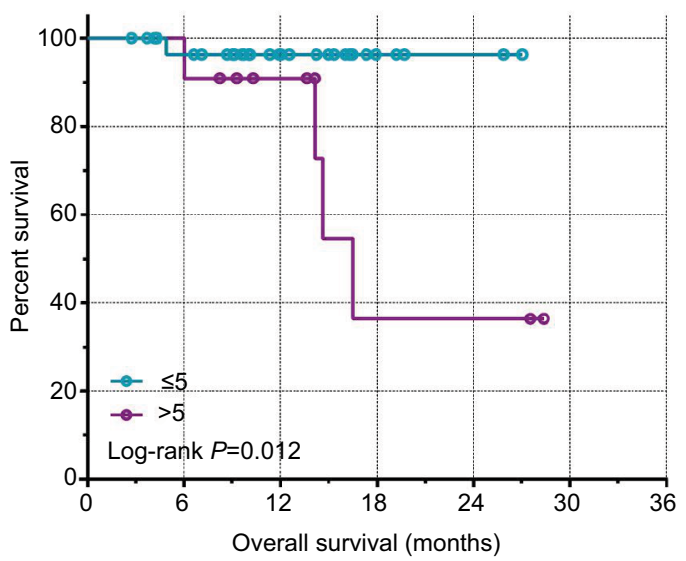

Figure 2 Kaplan-Meir survival curves for postoperative CTC counts.

Notes: (A) PFS with a CTC count cut-off of 2. (B) OS with a CTC count cut-off of 2. (C) PFS with a CTC count cut-off of 5 . (D) OS with a CTC count cut-off of 5. Abbreviations: CTC, circulating tumor cell; PFS, progression-free survival; OS, overall survival.

A

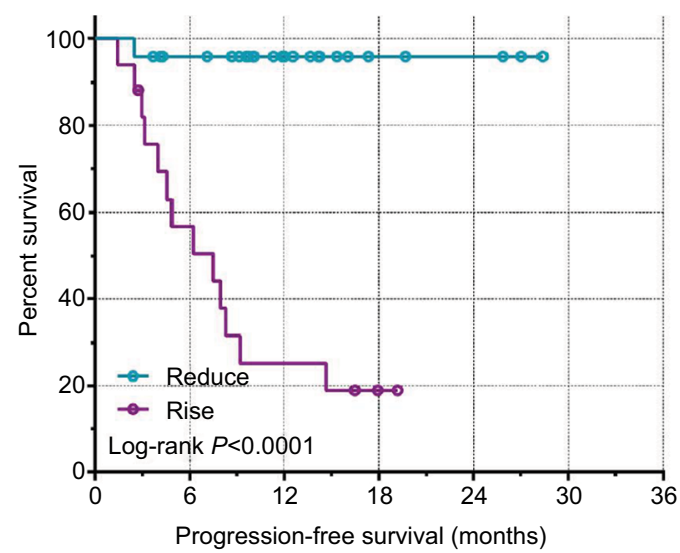

B

B

Figure 3 Kaplan-Meir survival curves for pre/postoperative change in CTC counts.

Notes: (A) PFS. (B) OS.

Abbreviations: CTC, circulating tumor cell; PFS, progression-free survival; OS, overall survival. 
Table 3 Cox regression analysis of associations between CTC counts and survival in patients with HBV-related HCC

\begin{tabular}{|c|c|c|c|c|c|}
\hline \multirow[t]{2}{*}{ Variables } & \multirow{2}{*}{$\begin{array}{l}\text { Patients } \\
(n=42)\end{array}$} & \multicolumn{2}{|l|}{ Overall survival } & \multicolumn{2}{|c|}{ Progression-free survival } \\
\hline & & $H_{R}^{a}(95 \% C l)$ & $P$-value ${ }^{a}$ & $\mathrm{HR}^{\mathrm{a}}(95 \% \mathrm{CI})$ & $P$-value ${ }^{a}$ \\
\hline \multicolumn{6}{|l|}{ Postoperative CTC counts } \\
\hline$\leq 2$ & 18 & 1 & & I & \\
\hline$>2$ & 24 & $1.16(0.047-28.25)$ & 0.379 & $8.72(1.40-54.17)$ & 0.020 \\
\hline \multicolumn{6}{|l|}{ Postoperative CTC counts } \\
\hline$\leq 5$ & 31 & 1 & & 1 & \\
\hline$>5$ & 11 & $15.65(0.80-304.64)$ & 0.069 & $6.89(1.64-29.00)$ & 0.009 \\
\hline \multicolumn{6}{|l|}{ CTC change } \\
\hline Reduce & 25 & 1 & & I & \\
\hline Rise & 17 & NA & 0.964 & $39.58(4.22-371.64)$ & 0.001 \\
\hline
\end{tabular}

Notes: ${ }^{a} \mathrm{HR}$ and $P$-value for Cox proportional hazard regression model. Adjustment by Tumor, Node, Metastases or Barcelona Clinic Liver Cancer stage, number of tumors, diameter of tumors, age, and gender.

Abbreviations: CTC, circulating tumor cell; AFP, alpha-fetoprotein; HBV, hepatitis B virus; HCC, hepatocellular carcinoma; HR, hazard ratio; Ref., reference; NA, not available.

receiving treatment after surgery. We found that patients with low postoperative CTC counts ( $\leq 2$ cells per $5 \mathrm{~mL}$ peripheral blood) had a significantly longer median PFS compared with patients with higher CTC counts (>2 cells per $5 \mathrm{~mL}$ peripheral blood), suggesting that a higher CTC count may be an independent marker of poor prognosis in terms of PFS and OS. Stebbing et $\mathrm{al}^{21}$ found that patients with $>100 \mathrm{CTCs}$ had a poor prognosis, with an average survival time of only 17 days. In the current study, seven patients with $>10$ CTCs per $5 \mathrm{~mL}$ showed disease progression during the observation period. This was consistent with previous reports. Patients with higher CTC counts may thus have higher recurrence risks and lower disease-free survival. ${ }^{22}$ We also compared preoperative and postoperative $\mathrm{CTC}$ counts in $\mathrm{HCC}$ patients and found a significant association between pre/postoperative change and prognosis, with patients with increased CTCs postoperatively having significantly longer median PFS compared with patients with a postoperative decrease in CTCs. This suggests that changes in CTC counts may be a more sensitive and accurate marker for estimating the prognosis of HCC patients. Preoperative examination and dynamic postoperative monitoring of CTCs in the peripheral blood may thus provide the basis for determining individualized treatment regimens for HCC patients in the future.

However, CTCs comprise a highly heterogeneous cell population, and the detection of a few more viable and aggressive CTCs and the analysis of the phenotypes and/or genotypes of CTCs may be more valuable than overall CTC counts. ${ }^{23}$ Targeted drugs are currently considered as a safe and effective way of treating HCC. However, identifying metastatic CTCs and determining the relevant signal transduction pathways represent the key to successful targeted drug therapy. ${ }^{24}$
In conclusion, these results demonstrate that postoperative CTC counts ( $>2$ and $>5$ ) and especially pre/postoperative changes in CTC counts may be independent prognostic indicators for PFS in patients with HBV-related HCC.

\section{Acknowledgments}

The authors thank Prof Minhao Peng, Kaiyin Xiao, Ya Guo, and Xigang Chen for providing this study with HCC samples. The authors thank Associate Prof Bin Chen and Zhixiong Su for their invaluable help to my study. This work was supported in part by the National Nature Science Foundation of China (No: 81560535), 2018 Innovation Project of Guangxi Graduate Education (No: JGY2018037 and YCBZ2018036), 2017 Guangxi Medical University Training Program for Distinguished Young Scholars, the Nature Sciences Found of Guangxi province of China (No: 2013GXNSFAA019138), and 2014 Project by Open Foundation of Guangxi Key Laboratory of Biological Targeting Diagnosis and Therapy Research.

\section{Disclosure}

The authors report no conflicts of interest in this work.

\section{References}

1. Torre LA, Bray F, Siegel RL, Ferlay J, Lortet-Tieulent J, Jemal A. Global cancer statistics, 2012. CA Cancer J Clin. 2015;65(2):87-108.

2. Khalaf N, Ying J, Mittal S, et al. Natural History of Untreated Hepatocellular Carcinoma in a US Cohort and the Role of Cancer Surveillance. Clin Gastroenterol Hepatol. 2017;15(2):273-281.

3. Bruix J, Sherman M, American Association for the Study of Liver Diseases. Management of hepatocellular carcinoma: an update. Hepatology. 2011;53(3):1020-1022.

4. Llovet JM, Hernandez-Gea V. Hepatocellular carcinoma: reasons for phase III failure and novel perspectives on trial design. Clin Cancer Res. 2014;20(8):2072-9. 
5. Sánchez Antolín G, García Pajares F, Pérez E, et al. Milan criteria versus San Francisco criteria in hepatocellular carcinoma: our center's experience. Transplant Proc. 2009;41(3):1012-3.

6. Kelley RK, Magbanua MJM, Butler TM, et al. Circulating tumor cells in hepatocellular carcinoma: a pilot study of detection, enumeration, and next-generation sequencing in cases and controls. BMC Cancer. 2015; 15(1):206.

7. Schulze K, Gasch C, Staufer K, et al. Presence of EpCAM-positive circulating tumor cells as biomarker for systemic disease strongly correlates to survival in patients with hepatocellular carcinoma. Int $J$ Cancer. 2013;133(9):2165-2171.

8. Mateo J, Gerlinger M, Rodrigues DN, de Bono JS. The promise of circulating tumor cell analysis in cancer management. Genome Biol. 2014;15(8): $1-10$.

9. Yang N, Hui L, Wang Y, Yang H, Jiang X. Overexpression of SOX2 promotes migration, invasion, and epithelial-mesenchymal transition through the Wnt/ $\beta$-catenin pathway in laryngeal cancer Hep-2 cells. Tumor Biology. 2014;35(8):7965-7973.

10. Friedlander TW, Premasekharan G, Paris PL, Back L. Looking back, to the future of circulating tumor cells. Pharmacol Ther. 2014;142(3):271-280.

11. Qiu MZ, Li ZH, Zhou ZW, et al. Detection of carcinoembryonic antigen messenger RNA in blood using quantitative real-time reverse transcriptase-polymerase chain reaction to predict recurrence of gastric adenocarcinoma. J Transl Med. 2010;8:107.

12. Reeh M, Effenberger KE, Koenig AM, et al. Circulating Tumor Cells as a Biomarker for Preoperative Prognostic Staging in Patients With Esophageal Cancer. Ann Surg. 2015;261(6):1124-1130.

13. Winters B, James A, Lee J, Kho J, Morrissey C, Wright J. Chemotherapeutic effects on circulating tumor cells in bladder cancer. International Journal of Urology. 2015;22(6):612-613.

14. Wu S, Liu Z, Liu S, Lin L, Yang W, Xu J. Enrichment and enumeration of circulating tumor cells by efficient depletion of leukocyte fractions. Clinical Chemistry and Laboratory Medicine. 2014;52(2):243-251.
15. Chinese Society of Liver Cancer, CA-CA, Chinese Society of Clinical Oncology, CA-CA, Liver Cancer Study Group CSo HCMA, Chinese Pathological Group of Hepatobiliary T. Expert consensus on the scheme of pathological diagnosis of primary liver cancer. Zhonghua Gan Zang Bing Za Zhi. 2011;19(4):254-256.

16. Scatton O, Chiappini F, Riou P, et al. Fate and characterization of circulating tumor cells in a NOD/SCID mouse model of human hepatocellular carcinoma. Oncogene. 2006;25(29):4067-4075.

17. Pantel K, Alix-Panabières C. Real-time liquid biopsy in cancer patients: fact or fiction? Cancer Res. 2013;73(21):6384-6388.

18. Yates DR, Rouprêt M, Drouin SJ, et al. Quantitative RT-PCR analysis of PSA and prostate-specific membrane antigen mRNA to detect circulating tumor cells improves recurrence-free survival nomogram prediction after radical prostatectomy. Prostate. 2012;72(12):1382-1388.

19. Sun Y-F, Xu Y, Yang X-R, et al. Circulating stem cell-like epithelial cell adhesion molecule-positive tumor cells indicate poor prognosis of hepatocellular carcinoma after curative resection. Hepatology. 2013;57(4):1458-1468.

20. $\mathrm{Xu} \mathrm{W}, \mathrm{Cao} \mathrm{L}, \mathrm{Chen} \mathrm{L}$. Isolation of circulating tumor cells in patients with hepatocellular carcinoma using an asialoglycoprotein receptorbased magnetic cell separation strategy. Clinical Cancer Research. 2011;17:3783-3793.

21. Stebbing J, Harding V, Urch CE, et al. The prognostic role of circulating tumor cells in heavily pretreated individuals with a low life expectancy. Future Oncol. 2014;10(16):2555-2560.

22. Liu Z, Guo W, Zhang D, et al. Circulating tumor cell detection in hepatocellular carcinoma based on karyoplasmic ratios using imaging flow cytometry. Sci Rep. 2016;6:39808.

23. Zhang Y, Li J, Cao L, Xu W, Yin Z. Circulating tumor cells in hepatocellular carcinoma: detection techniques, clinical implications, and future perspectives. Semin Oncol: Elsevier; 2012;39(4):449-460.

24. van de Stolpe A, den Toonder J. Circulating Tumor Cells: What Is in It for the Patient? A Vision towards the Future. Cancers. 2014;6(2):1195-1207.
Cancer Management and Research

\section{Publish your work in this journal}

Cancer Management and Research is an international, peer-reviewed open access journal focusing on cancer research and the optimal use of preventative and integrated treatment interventions to achieve improved outcomes, enhanced survival and quality of life for the cancer patient. The manuscript management system is completely online and includes

\section{Dovepress}

a very quick and fair peer-review system, which is all easy to use. Visit http://www.dovepress.com/testimonials.php to read real quotes from published authors. 